\title{
Einsatz standardisierter Patienten im Psychologiestudium: Von der Forschung in die Praxis
}

\author{
Franziska Kühne Ulrike Maaß Florian Weck \\ Abteilung für Klinische Psychologie und Psychotherapie, Universität Potsdam, Potsdam, Deutschland
}

\author{
Schlüsselwörter \\ Training · Ausbildung · Psychotherapie · Evidenzbasierte \\ Versorgung
}

\section{Zusammenfassung}

Hintergrund: Im Rahmen des reformierten Psychotherapeutengesetzes wird eine stärkere Praxisorientierung in der klinisch-psychologischen Lehre und in der Prüfung psychotherapeutischer Kompetenzen verankert. Hierbei sollen Studierende durch die Interaktion mit standardisierten Patient*innen (SP) therapeutische Kompetenzen erwerben und demonstrieren. Fragestellung: Das Ziel des vorliegenden Beitrags ist es, eine evidenzbasierte Umsetzung dieser neuen Lehr- und Prüfungsformate zu unterstützen, indem bisherige Forschungsbefunde zum Einsatz von SP dargestellt und Bereiche, in denen weitere Forschung notwendig ist, aufgezeigt werden. Ergebnisse: Empirische Befunde zeigen, dass SP psychische Störungen authentisch darstellen können. Voraussetzung dafür sind beispielsweise die Auswahl geeigneter SP, detaillierte Rollenanleitungen, spezifisches Training, Feedback und Nachschulungen. Auch wenn einige Forschungsfragen, wie zur vergleichenden Wirksamkeit des Einsatzes von SP, noch unbeantwortet sind, lassen sich praktische Implikationen für SP-Programme in Lehre, Prüfung und Forschung ableiten, die in einem Ablaufschema dargestellt werden. Schlussfolgerungen: Der Einsatz von SP bietet großes Potenzial für die klinisch-psychologische Lehre und Ausbildungsforschung. Um den Einsatz von SP an ande- ren Standorten zu unterstützen, werden Beispielmaterialien (z.B. Rollenanleitung) in den elektronischen Supplementen (siehe www.karger.com/doi/10.1159/000509249 für alle Supplemente) zum Artikel zur Verfügung gestellt.

(c) 2020 S. Karger AG, Basel

\author{
Standardized Patients in Clinical Psychology: From \\ Research to Practice
}

\section{Keywords \\ Training · Education · Psychotherapy · Evidence-based care}


onstrate that SPs are able to present mental disorders au thentically. Prerequisites are, among others, the selection of suitable SPs, detailed role scripts, specific training, feedback, and corrective training. Although some research questions, including the comparative effectiveness of SPs, remain unanswered, practice implications for using SPs in education, examination, and research can be drawn. These implications are illustrated schematically. Conclusions: The use of SPs has large potential for education in clinical psychology and for research on psychotherapy training. With a view to encouraging the widespread use of SPs, we provide exemplary materials (e.g. role script) within the online supplements (see www. karger.com/doi/10.1159/000509249 for all online suppl. material). ○) 2020 S. Karger AG, Basel

\section{Verabschiedung des Psychotherapeutengesetzes}

Mit dem Gesetz zur Reform der Psychotherapeutenausbildung [Gesetz zur Reform der Psychotherapeutenausbildung (PsychThG), 2019] ist nun auch in Deutschland eine stärkere Praxisorientierung in der klinisch-psychologischen Lehre und in der Prüfung therapeutischer Kompetenzen verbunden. In der aktuellen Approbationsordnung für Psychotherapeutinnen und Psychotherapeuten [Approbationsordnung für Psychotherapeutinnen und Psychotherapeuten (PsychThApprO), 2020] ist verankert, dass die Approbationsprüfung zum Abschluss des Masterstudiums Psychotherapie aus einer mündlich-praktischen Fallprüfung und einer anwendungsorientierten Parcoursprüfung in fünf Kompetenzbereichen bestehen wird. Als Kompetenzbereiche wurden die Themen Patientensicherheit, therapeutische Beziehungsgestaltung, Diagnostik, Patienteninformation und -aufklärung sowie leitlinienorientierte Behandlungsempfehlungen vorgeschlagen [PsychThApprO, 2020]. In jedem Themenfeld sollen "Simulationspatient*innen" eingesetzt und Prüflinge hinsichtlich ihrer Leistungen bei der Durchführung bewertet werden. Das Institut für medizinische und pharmazeutische Prüfungsfragen (IMPP) könnte beauftragt werden, Prüfungsaufgaben für die Themenbereiche $\mathrm{zu}$ entwickeln, Bewertungsschlüssel und Musterlösungen vorzuschlagen und die Prüfungen auszuwerten [Deutsche Gesellschaft für Psychologie (DGPs), 2020]. Außerdem könnte das IMPP sowohl Simulationspatient*innen als auch Prüfer*innen, die auf Vorschlag der Universitäten bestellt werden, trainieren. Es wird also zu einer Reihe von Veränderungen kommen, wobei Ausbildungs- und Prüfungsformen stärker an die medizinische Ausbildung angepasst werden und einen deutlicheren Handlungsbezug aufweisen sollen [Willutzki et al., 2015].
Das Ziel des vorliegenden Beitrags ist es, empirische Befunde zusammenzutragen, die eine evidenzbasierte Etablierung der neuen Lehr- und Prüfungsformate gewährleisten. Neben Empfehlungen zur praktischen Implikation werden offene Fragen aufgezeigt, zu denen noch Forschungsbedarf besteht.

\section{Simulationspatient*innen in der medizinischen Ausbildung}

Bereits seit den 1960er Jahren werden simulierte Rollenspiele in der medizinischen Ausbildung eingesetzt [Barrows et al., 1993]. Dabei werden Begriffe unterschiedlich verwendet: "Simulationspatient*innen" sind (meist trainierte) Laien, die ein Beschwerdebild zu Lehr- oder Prüfungszwecken präsentieren [Adamo, 2003; Sheen et al., 2015; Pheister et al., 2017]. Bei "standardisierten Patient*innen" setzt man voraus, dass sie ein Beschwerdebild wiederholt und konsistent darbieten [Barrows, 1993; Adamo, 2003] und Lerner*innen bei Bedarf Feedback geben können [Pheister et al., 2017]. Werden Berufsschauspieler*innen eingesetzt, nutzt man mitunter den Begriff "Schauspielpatient*innen" [Partschefeld et al., 2020]. Die oben genannten Bezeichnungen werden allerdings uneinheitlich und oft auch synonym verwendet. Da unser Fokus darauf liegt, die wiederholte und konsistente Falldarstellung durch trainierte Laien zu beschreiben, nutzen wir den Begriff "standardisierte Patient*innen" (SP).

Der Einsatz von SP bringt zahlreiche Vorteile mit sich. Allen voran ist zu nennen, dass nicht nur Faktenwissen (z.B. über Störungsbilder und deren Behandlung), sondern vor allem Handlungskompetenzen ("Zeigen, wie es geht") in kontrollierten Situationen trainiert und geprüft werden können [Weck et al., 2019]. Dadurch werden nachfolgende Interaktionen mit realen Patient*innen in einer sicheren Lernumgebung vorbereitet [Barrows, 1993; Weck et al., 2019]. Der Einsatz von SP ist ethisch weniger bedenklich und leichter organisierbar als der Einbezug von realen Patient*innen [Voderholzer, 2007; Imel et al., 2014; Pheister et al., 2017]. Lerner*innen erhalten handlungsbezogenes Feedback schon im Studium und nicht erst, wenn Fehler oder Schwierigkeiten im Therapieprozess auftreten [Hodges et al., 2002].

Im Gegensatz zur Variabilität, die Patientenfällen eigen ist, ermöglichen SP eine stringentere Ausrichtung an Gütekriterien, d.h. reliablere, validere und fairere Kompetenzprüfungen [Yudkowsky, 2002]. Es können gezielt repräsentative Symptome oder zentrale Interventionen ausgewählt werden, die jeder Prüfling in gleicher Weise im Rollenspiel bearbeitet [Barrows, 1993; Fairburn und Cooper, 2011; Pheister et al., 2017]. SP können zudem wiederholt Interaktionen mit einer Person, aber 
auch mit einer großen Anzahl von Lerner*innen, spielen und sie können die Schwere ihrer Darstellung je nach Aufgabenstellung gezielt variieren [Barrows, 1993; Fairburn und Cooper, 2011]. Auf SP wird insbesondere dann zurückgegriffen, wenn diagnostische und Gesprächsführungskompetenzen trainiert oder der Umgang mit seltenen, mit emotional oder therapeutisch schwierigen oder mit ethisch sensiblen Situationen erprobt werden soll [Barrows, 1993; Eagles et al., 2007; für eine Übersicht siehe Eckel et al., 2014]. Zukünftige Forschung könnte dazu beitragen darzulegen, in welchen Themenbereichen der Einsatz von SP besonders bzw. weniger hilfreich ist.

In der medizinischen Ausbildung werden SP vielfach auch zur Beurteilung klinischer Kompetenzen in sogenannten Parcoursprüfungen herangezogen [Barrows, 1993]. Eine Parcoursprüfung ist ein spezielles Prüfungsformat, in dem Prüflinge verschiedene Stationen durchlaufen, die realen klinischen Situationen nachempfunden sind [Sheen et al., 2015]. Durch die Aneinanderreihung von bezüglich Inhalt und Schwere standardisierten Prüfungsstationen wird eine reliablere Kompetenzeinschätzung als bei der Beobachtung einer Einzelsituation angestrebt [Barrows, 1993; Hodges et al., 2002; Yudkowsky, 2002; Adamo, 2003; Imel et al., 2014]. Auch für die Parcoursprüfung werden unterschiedliche Termini genutzt (Objective Structured Clinical Examination [OSCE], clinical skills examination, performance-based assessment etc.), da der Grad der Strukturierung der Aufgabenstellungen, die Dauer pro Station (wenige Minuten bis zu über eine Stunde) und die Anzahl der zu bewertenden Fertigkeiten stark variieren. Beispielsituationen sind dabei:

- die Behandlung eines Behandlungsfalls von der Eingangsdiagnostik bis zum Abschlussgespräch an aufeinander folgenden Stationen oder über mehrere Termine hinweg,

- das Anwenden unterschiedlicher diagnostischer und therapeutischer Inhalte an verschiedenen Stationen,

- die Interaktion mit SP an einigen Stationen, während an anderen Stationen Prüfungen ohne SP stattfinden, oder

- das Stellen von Prüfungsfragen im Anschluss an ein Rollenspiel mit SP [Barrows, 1993; Coyle et al., 1998; Hodges et al., 2002; Yudkowsky, 2002; Adamo, 2003]. Trainings und Parcoursprüfungen mit SP sind aufgrund ihrer Vorteile bereits jetzt ein wichtiger Bestandteil der medizinischen Ausbildung, auch in den Fächern Neurologie und Psychiatrie [Barrows, 1993; Yudkowsky, 2002; Eagles et al., 2007; Wündrich et al., 2017]. Wurde in der klinisch-psychologischen Ausbildung und Forschung bis dato seltener auf SP zurückgegriffen [Sheen et al., 2015; Edwards et al., 2016; Kühne et al., 2018], wird sich dies mit der beschlossenen Gesetzesänderung ver- mutlich wandeln, wodurch der Einsatz von SP in Ausbildung und Forschung stärker in den Vordergrund rücken wird (Abb. 1).

\section{Simulationspatient*innen in Psychologiestudium und Psychotherapieausbildung}

Um erfahrungsbasierte und praxisorientierte Lernumgebungen zu schaffen und Lehre und Prüfungen kompetenzbasiert zu gestalten, ist der Einbezug von SP auch in der Ausbildung von Psychotherapeut*innen lohnenswert [Ehrenthal et al., 2019]. Im Vergleich zur breiteren Literaturbasis zur Gestaltung von SP-Programmen in Lehre und Forschung fällt die Anzahl methodisch hochwertiger empirischer Studien zur Effektivität dieser Programme ab. Im nächsten Absatz werden beispielhaft einige wichtige Studien zu Effekten von SP vorgestellt. In den darauffolgenden Abschnitten (3.1. bis 3.8.) werden den einzelnen Schritten beim Aufbau von SP-Programmen entsprechend spezifischere empirische Ergebnisse beleuchtet.

Sheen et al. [2015] und Yap et al. [2012] berichten, dass Psychologiestudent*innen in einem Master- und einem Doktorandenprogramm Parcoursprüfungen subjektiv als Stress und Angst auslösend, aber auch als realistische Annäherungen an die klinische Praxis und als positive Lerngelegenheiten erlebten. Schließlich kommen Eckel et al. [2014] in ihrer Übersichtsarbeit zum Einsatz von SP in psychosozialen Studienfächern zu dem Schluss, dass Student*innen SP-Interaktionen ganz überwiegend als authentisch und positiv erleben und einen subjektiven Lernzuwachs wahrnehmen.

Entsprechend profitierten Ausbildungskandidat*innen $(n=20)$ mit Vertiefungsrichtung Tiefenpsychologie sowohl subjektiv als auch aus Sicht trainierter Urteiler*innen von Rollenspielen mit SP [Nikendei et al., 2019]. Allerdings wurden in dieser Studie unterschiedliche Trainingsaspekte kombiniert, sodass der Rückschluss auf den Einsatz von SP nicht eindeutig möglich ist. Einer weiteren Untersuchung zufolge hatten 29 Ausbildungskandidat*innen der Kinderund Jugendpsychotherapie nach einem 16 Unterrichtsstunden umfassenden Training mit SP aus Sicht dieser SP und aus Perspektive zweier Fremdurteiler*innen signifikant ausgeprägtere therapeutische Fertigkeiten und zeigten mehr Empathie als davor [Partschefeld et al., 2013]. Eine Limitation dieser Studie war jedoch, dass kein Vergleich mit einer Kontrollgruppe stattfand.

Um evidenzbasierte Empfehlungen zur Gestaltung von klinisch-psychologischer Lehre geben zu können, ist es unerlässlich, dass sich die Ausbildungsforschung zukünftig noch gezielter mit den Effekten von SP-Programmen beschäftigt. Nichtsdestotrotz lassen sich Empfehlungen für den Einsatz von SP aus der aktuellen 


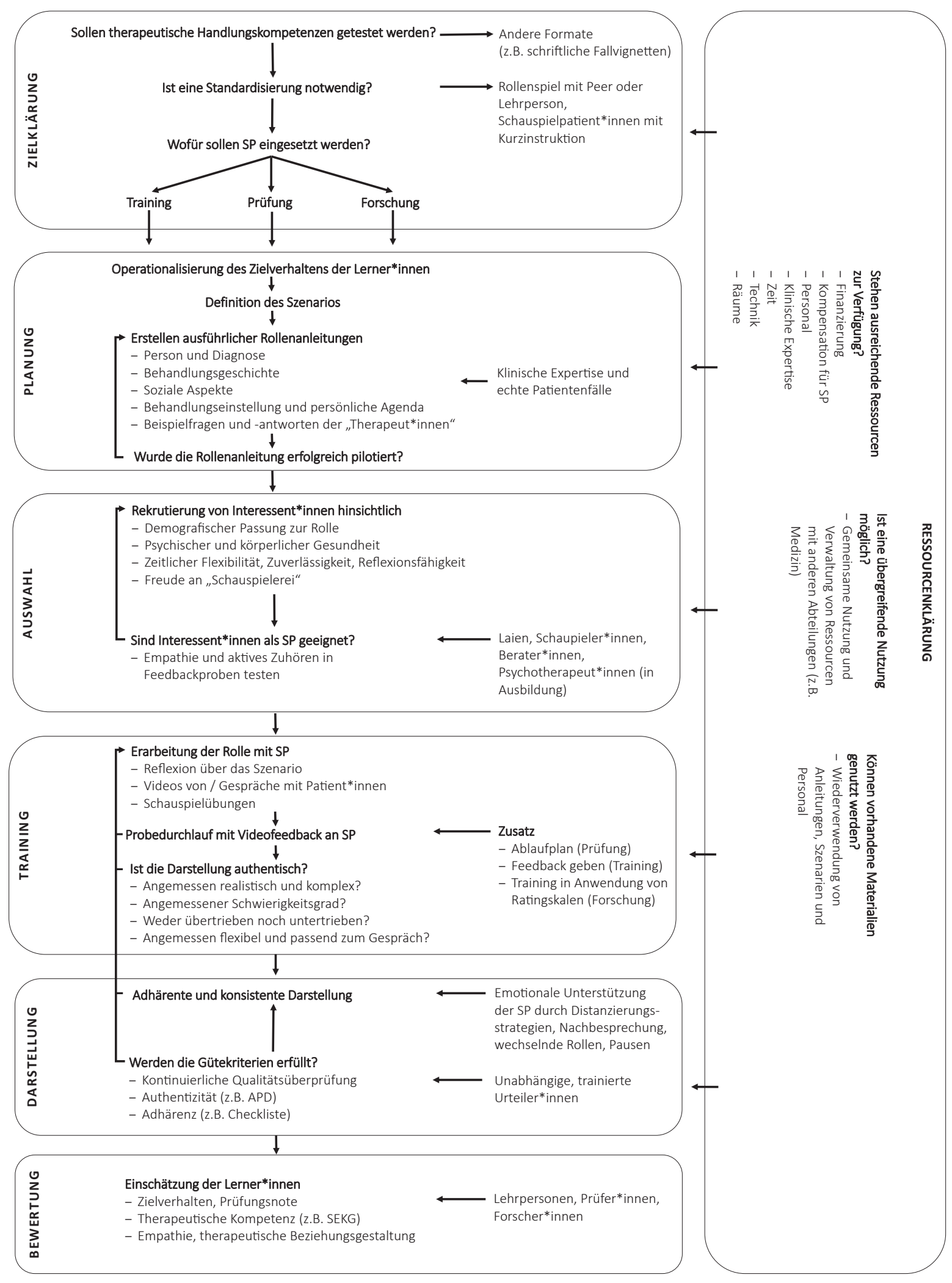

Abb. 1. Zentrale Schritte beim Einbezug von SP in Lehre und Forschung [siehe auch Kühne et al., 2018]. 
Literatur ableiten, die wir im Folgenden anhand der Schritte zum Aufbau eines SP-Programms zusammenfassen.

\subsection{Welche Ziele hat der Einsatz von SP?}

Bevor ein SP-Programm gestaltet werden kann, sollte spezifiziert werden, warum in ein solches investiert werden soll, und welcher Vorteil gegenüber anderen Formaten wie der schriftlichen Bearbeitung von Fallvignetten oder dem Üben in Peer-Rollenspielen erwartet wird [Kühne et al., 2018]. Da das Ziel eines SP-Programms zwangsläufig dessen konkrete Ausgestaltung bestimmt, sollte zunächst spezifiziert werden, wofür SP eingesetzt werden sollen: für die Lehre (formative Evaluation), zur Leistungsprüfung (summative Evaluation) oder in der Forschung. In der Lehre kann es wichtiger sein, dass SP den Teilnehmer*innen Feedback geben, während dieser Aspekt in einer Prüfung unter Umständen wegfallen kann. Dagegen ist es bei einer Prüfung oder in der Forschung wichtig, dass SP ihre Rollen möglichst adhärent und konsistent darstellen, um Vergleichbarkeit zu gewährleisten.

\subsection{Welche Ressourcen stehen zur Verfügung?}

Der gewissenhafte Einsatz von SP ist zeit- und kostenintensiv [Pheister et al., 2017]. So werden entsprechend viele Räume, aber auch Vollzeit-Personal benötigt, das beispielsweise SP rekrutiert, trainiert, Einsätze plant und überwacht, Videoaufnahmen gewährleistet, Materialien zur Verfügung stellt, Daten sichert, Feedback gibt, Urteiler*innen und Prüfer*innen trainiert und begleitet und Ergebnisse auswertet [Adamo, 2003]. Die Einführung eines SP-Programms kann daher auf Seiten der Organisation und des Personals auf Widerstände stoßen, weshalb Vor- und Nachteile des Einsatzes von SP und die erforderlichen Ressourcen frühzeitig kommuniziert werden sollten [Voderholzer, 2007]. Beispiele für Kostenkategorien und Argumente, um die Finanzierung zu sichern, führen Hodges et al. [2002] auf. Die Analogie zu anderen Fachgebieten, wie der Luft- und Raumfahrt oder zu Großschadensfällen, bei denen simulationsbasierte Trainings weithin akzeptiert sind [Adamo, 2003], könnte ebenfalls zur Akzeptanzförderung genutzt werden. Für einen ressourcensparenden Einsatz kann über einen gemeinsamen SP-Pool, ein gemeinsames Training, geteilte Materialien oder ein gesamtes SP-Programm über Abteilungen oder Einheiten hinweg nachgedacht werden [Eagles et al., 2007].

\subsection{Wie können Rollenanleitungen entwickelt werden?}

Wurde das Ziel des SP-Programms festgelegt und stehen genügend Ressourcen für die Implementierung zur Verfügung, besteht einer der ersten Schritte in der Ent- wicklung von Rollenanleitungen für SP und von Instruktionen für Lerner*innen. Obwohl einige Autor*innen freiere Rollenspiele bevorzugen, um spontane Interaktionen zu unterstützen, sprechen sich andere für das Erstellen detaillierter Szenarios aus [Kühne et al., 2018]. Auch von zu strengen Szenarien wird abgeraten, da nicht alle Antworten der Lerner*innen im Vorhinein bekannt seien [Pheister et al., 2017]. Wir empfehlen das Erstellen ausführlicher Rollenanleitungen, wie beispielsweise eine Untersuchung unserer Arbeitsgruppe zu dieser Frage zeigt: Personen, die vor dem Rollenspiel eine ausführliche Rollenanleitung gelesen hatten, wurden sowohl aus Perspektive einer approbierten Therapeutin als auch aus Sicht zweier trainierter Urteiler*innen als authentischer bewertet als SP, die nur eine sehr basale Beschreibung erhielten [Ay et al., 2020a].

Um Realitätsnähe herzustellen, können Rollenanleitungen ausgehend von echten Patientenfällen entwickelt werden [Pheister et al., 2017]. Parallel dazu sollten die Lern- und Prüfungsziele [Voderholzer, 2007] bzw. die von den Prüflingen erwarteten Verhaltensweisen operationalisiert werden [z.B. "Fragt nach Alkoholkonsum"; Hodges et al., 2002]. Es wird empfohlen, SP detaillierte Angaben zur Person, Diagnose, Behandlungsgeschichte, zu sozialen Aspekten, der Einstellung des zu spielenden Charakters zur Behandlung, zu seiner Agenda (z.B. Wunsch nach Medikation) sowie Beispielfragen und -antworten von Lerner*innen zur Verfügung zu stellen [Voderholzer, 2007; Pheister et al., 2017]. Trotzdem ist es wichtig, das Szenario allgemeinverständlich und an den Lehr- und Prüfungszielen orientiert zu formulieren, um unnötige Details zu vermeiden [Voderholzer, 2007]. In die Entwicklung der Rollenanleitungen sollten Fachexpert*innen einbezogen werden, es können aber auch SP und reale Patient*innen mitwirken [Kühne et al., 2018]. Ein Beispiel einer Rollenanleitung für eine depressive Störung findet sich im Online-Supplement 1.

\subsection{Wie wählt man geeignete SP aus?}

Die umsichtige Auswahl von SP ist besonders für deren Einsatz in Prüfungen entscheidend [Hodges et al., 2002], da mit vertraulichen Daten, wie den Prüfungsergebnissen und dem Verhalten der Prüflinge, umgegangen werden muss [Voderholzer, 2007]. SP werden zunächst aufgrund ihrer Passung zur geplanten Rolle beispielsweise auf Basis demografischer Merkmale, ihrer Vorbildung oder Schauspielerfahrung ausgewählt [Adamo, 2003]. Neben Laien können auch Schauspieler*innen [Adamo, 2003], erfahrene Berater*innen und Psychotherapeut*innen [Coyle et al., 1998], Kandidat*innen in fortgeschrittener Ausbildung [Pheister et al., 2017] oder Mitarbeiter*innen [Hodges et al., 2002] als SP einbezogen werden. Nehmen SP nicht als Freiwillige gegen eine Teilnahmevergütung, sondern als Angestellte teil, so kann von ihnen noch stärker 
eingefordert werden, dass sie alle Qualitätsstandards gewissenhaft einhalten [Adamo, 2003], allerdings ist ihre Teilnahme nicht zwangsläufig ressourcensparender [Hodges et al., 2002].

Uneinigkeit besteht darüber, ob SP auch auf eigene Erkrankungserfahrungen zurückgreifen sollten, und wenn ja, in welchem Ausmaß [Barrows, 1993; Adamo, 2003; Pheister et al., 2017]. Aus unserer Sicht ist der Bezug auf eigene Erkrankungserfahrungen bei körperlichen Erkrankungen eher denkbar, zumal, wenn tatsächliche Vorbefunde oder Behandlungsfolgen, wie beispielsweise Narben, in die Rollendarstellung einbezogen werden sollen [Barrows, 1993; Voderholzer, 2007]. Schwerwiegende aktuelle Beschwerden sollten dennoch vorab ausgeschlossen werden [beispielsweise anhand eines Anamnesebogens; Voderholzer, 2007].

Bei der Darstellung psychischer Störungen können eigene Erkrankungserfahrungen dagegen $\mathrm{zu}$ einer $\mathrm{zu}$ starken Vermischung von Rolle und eigenem Erleben und zu einer Belastung der SP führen [Kühne et al., 2018]. Damit SP geschützt werden und nicht mit Lerner*innen in Interessenskonflikte geraten, sind unsere SP körperlich und psychisch gesunde Studierende, die zumeist nicht Psychologie studieren. Außerdem haben wir bei der Auswahl auf Empathie und Reflexionsfähigkeit, Freude an schauspielerischer Tätigkeit und wissenschaftlicher Arbeit, zeitliche Flexibilität und auf Zuverlässigkeit geachtet. Voderholzer [2007] weist darauf hin, dass auch die Einsicht in eigene Verhaltensweisen gegeben sein sollte. Adamo [2003] empfiehlt darauf zu achten, dass SP sich auf unterschiedliche Lerner*innen einstellen, die Darstellung emotional bewältigen, ihr Verhalten anhand von Rückmeldungen der Lehrpersonen anpassen, aktiv zuhören und sich vom Training ausgehend auf unterschiedliche Anwendungsbereiche einstellen können. Rollenspiel- und Feedbackproben während des Auswahlprozesses sind Mittel, um die Eignung von SP verhaltensnah zu beurteilen [Voderholzer, 2007]. Die Forschung könnte der Frage nachgehen, welche Grenzen es bei der Simulation welcher psychischen Störungen gibt.

\subsection{Wie trainiert man SP?}

Dem Training von SP wird übereinstimmend eine zentrale Rolle zugeschrieben [Hodges et al., 2002; Adamo, 2003; Voderholzer, 2007; Kühne et al., 2018]. Da Ziele und Ressourcen von SP-Programmen variieren, existiert kein etablierter und übergreifender Standard, wieviel Training notwendig ist [Adamo, 2003]. Hodges et al. [2002] empfehlen pro SP ein 4- bis 6-stündiges Training. Dieses solle die Reflexion über das Szenario, Videos von oder Gespräche mit Patient*innen, Schauspielübungen und Probedurchläufe enthalten [Hodges et al., 2002; Yudkowsky, 2002]. Der Bezug zu eigenen Gefühlen und Lebenserfahrungen und die Möglichkeit, mitent- scheiden zu dürfen, welche Rolle gespielt wird, kann helfen, dass sich SP in die Symptomatik hineinversetzen können [Kühne et al., 2018]. Forschungsbedarf besteht hinsichtlich der Frage, welche Trainingsbestandteile essentiell und welche vernachlässigbar sind.

Das Hauptziel des Trainings ist die valide Präsentation des Beschwerdebilds [Adamo, 2003]. SP sollten Symptome nicht zu übertrieben oder zu stark auf eigenen Erlebnissen beruhend präsentieren, Fälle nicht zu geradlinig oder lehrbuchartigen darstellen, Symptomatik und Gestik sollten zur Situation passen, und sie sollten die Rollenspiele nicht zu leicht oder schwer für die Lerner*innen gestalten [Eagles et al., 2007; Wuendrich et al., 2012]. Um die Darstellung dementsprechend zu präzisieren, sind Proberollenspiele mit Videofeedback ein gutes Mittel. Außerdem ist es hilfreich, wenn SP nicht nur die Szenarios, sondern auch begleitende Informationen, beispielsweise zum Feedback geben (siehe 3.7), zur Vorbereitung auf die Rolle oder zum Ablauf des Prüfungstages in Form eines Handbuchs erhalten [UKE, 2020]. Zur Vorbereitung gehört auch, Lehrpersonal für die Arbeit mit SP zu schulen [Hodges et al., 2002; Kienle und Peters, 2016], damit sie SP begleiten und bei Problemen frühzeitig intervenieren können.

\subsection{Was ist bei der Darstellung psychischer}

Erkrankungen zu beachten?

Das Ziel des SP-Programms bestimmt konsequenterweise auch, ob die Darstellung eher improvisiert (gegebenenfalls in der Lehre) oder eher standardisiert sein soll (zu Prüfungs- und Forschungszwecken [Kühne et al., 2018]). Das Szenario bietet zwar den Rahmen, aber das Training sollte SP darauf vorbereitet haben, dass ein gewisser Grad an Improvisation unumgänglich ist, um auf das Verhalten der Lerner*innen angemessen reagieren $\mathrm{zu}$ können [Pheister et al., 2017].

Psychische Erkrankungen zu simulieren kann herausfordernd für SP sein [Yudkowsky, 2002; Eagles et al., 2007; Voderholzer, 2007]. Es ist wichtig, mit SP darüber zu sprechen und vorab Strategien zu ihrer Entlastung zusammenzutragen [Hodges et al., 2002]. Probedurchläufe, Beratung und Supervision für SP, wechselnde Rollen, regelmäßige Pausen oder feste Nachbesprechungen können entlasten [Kühne et al., 2018]. Außerdem sollte besprochen werden, wie viele Interaktionen pro Tag nacheinander zu bewältigen sind. All dies ist entscheidend, da die Art der Darstellung der SP mitbestimmt, wie sich Lerner*innen in die Situation einfühlen können, wie Gütekriterien ausfallen und damit auch, wie das Lern- oder Prüfungsergebnis sein wird [Wuendrich et al., 2012; Kühne et al., 2018].

\subsubsection{Wie können Fälle authentisch dargestellt} werden?

Limitationen des Einsatzes von SP im Rahmen der Psychotherapieausbildung werden mitunter darin gese- 
hen, dass das "einzigartige interpersonelle Geschehen" nicht simuliert werden könne [Brenner, 2009] oder dass Falldarstellungen zu vereinfachend und wenig repräsentativ für die klinische Praxis seien [Muse und McManus, 2013]. Mehrere Untersuchungen gingen daher der Frage der Authentizität - d.h. der Unmöglichkeit SP und reale Patient*innen zu unterscheiden [Wuendrich et al., 2012] - nach.

Werden SP als unauthentisch erlebt, so liegt dies meist an einer vereinfachten oder übertriebenen Symptomdarstellung, an einer wenig überzeugend dargebotenen Lebensgeschichte oder an Problemen, sich auf begleitende technische Unterstützung wie Videoaufnahmen einzustellen [Eckel et al., 2014]. Je öfter SP einen Fall darstellen, desto eher kann es dazu kommen, dass sie eine Lehrposition einnehmen, Lerner*innen Hinweise geben und ihre Rolle verlassen [Adamo, 2003]. Dies sollte gemeinsam reflektiert und nach Lösungen gesucht werden. Wichtig ist auch, dass fortlaufend geprüft wird, ob SP ihre Rollen noch authentisch spielen und gegebenenfalls nachzuschulen.

Die Mehrzahl der bisherigen Befunde zeigt entgegen mancher Vermutung, dass mit adäquatem Training eine authentische Falldarstellung gelingen kann [Yudkowsky, 2002; Eckel et al., 2014]. In einer Videostudie wurden SP von erfahrenen Psychiater*innen in 61\% der Fälle als reale Patient*innen (!) identifiziert; reale Patient*innen wurden in $94 \%$ der Fälle als solche erkannt [Wuendrich et al., 2012]. Patient*innen wurden als authentischer wahrgenommen, SP-Fälle dagegen als typischer und einprägsamer, was dem Ziel, fokussierte Lerngelegenheiten zu schaffen, genau entspricht.

Zur Einschätzung der Authentizität von Patientendarstellungen haben wir ein Kurzinstrument entwickelt [AyBryson et al., 2020; erhältlich auf Anfrage von der Korrespondenzautorin]. In der Instruktion wird nicht offengelegt, ob SP oder reale Patient*innen eingeschätzt werden sollen; Beispielitems lauten: "Die Person beschreibt ihre Erkrankung insgesamt überzeugend" oder "Das Verhalten der Person ist dem Erkrankungsbild angemessen". In einer zugehörigen Studie wurden Psychotherapeut*innen in Ausbildung sechs Videos präsentiert, wobei sie SP und reale Patient*innen nicht signifikant voneinander unterscheiden konnten [Ay et al., 2020b]. Hierzu besteht allerdings weiterer Forschungsbedarf unter Einbezug größerer Stichproben und in Ausweitung auf unterschiedliche Störungsbilder.

\subsubsection{Wie gelingt eine adhärente Falldarstellung?}

Adäquates Training und die fortlaufende Prüfung der Fallpräsentation sind nicht nur für eine authentische Falldarstellung nötig, sondern auch dafür, dass die Darstellung dauerhaft in gleicher Weise (d.h. konsistent adhärent) dargestellt wird. Um die Adhärenz der Darstellung zu prüfen, kann beispielsweise kontrolliert werden, ob SP die im Szenario beschriebenen Verhaltensweisen umsetzen bzw. ob sie Verhaltensweisen zeigen, die nicht im Szenario vorgesehen sind [Waltz et al., 1993; Edwards et al., 2016]. SP sollten den Charakter wie vorgesehen darstellen, sich z.B. angemessen kooperativ zeigen und im Rollenspiel Informationen in passendem Umfang preisgeben [Edwards et al., 2016]. Anhand von Videoaufnahmen werden Gütekriterien (z.B. Inhaltsvalidität und Inter-Rater-Reliabilität) geprüft und gegebenenfalls Abweichungen sichtbar, worauf Nachschulungen initiiert werden sollten [Edwards et al., 2016; Pheister et al., 2017]. Beispielitems zur Adhärenzprüfung sind Online-Supplement $2 \mathrm{zu}$ entnehmen.

\subsection{Was ist beim Feedbackgeben zu beachten?}

Neben Lehrpersonen oder anderen Trainingsteilnehmer*innen können auch SP den Lerner*innen Feedback geben [z.B. "Als Ihr Patient fühlte ich mich umsorgt, aber ich hatte Probleme, Sie zu verstehen, weil Sie zu viele Fachworte benutzt haben [...]"; Pheister et al., 2017, S. 115, Übers. d. Autoren]. Diese Form der Rückmeldung, die von realen Patient*innen nicht im selben Ausmaß erwartet werden kann, bietet Lerner*innen eine besondere Lerngelegenheit, vorausgesetzt das Feedback wird angemessen und wertschätzend gegeben [Coyle et al., 1998].

Obwohl es sein kann, dass sich die Rückmeldungen von SP und echten Patient*innen unterscheiden, wird angenommen, dass dieses Feedback neben der Verbesserung von technischen Fertigkeiten zum Aufbau von Empathie und dem Verbessern der Beziehungsgestaltung beiträgt [Pheister et al., 2017]. Außerdem wird davon ausgegangen, dass Personalressourcen gespart werden können, wenn SP Lerner*innen klar umgrenztes Feedback im Anschluss an jede Interaktion geben [Voderholzer, 2007]. Aus unserer Perspektive sollten Rückmeldungen zur therapeutischen Kompetenz nicht von SP gegeben werden, sondern dem Lehrpersonal vorbehalten bleiben.

In jedem Fall sollten SP ein spezielles Training dazu erhalten, wie ein konstruktives Feedback aussieht [Adamo, 2003; UKE, 2020]. Feedback kann verbal oder schriftlich, frei oder anhand einer Checkliste, direkt im Anschluss oder anhand einer Audio- oder Videoaufnahme gegeben werden [Adamo, 2003; Pheister et al., 2017]. Insgesamt ist es hilfreich, wenn Feedback-Protokolle genutzt werden und Feedbacks beispielsweise von einer Lehrperson fortlaufend geprüft werden, um auch hier gegebenenfalls nachschulen zu können [Kühne et al., 2018].

\subsection{Wie kann man Kompetenzen erfassen?}

Kompetenzen können nur dann reliabel geprüft werden, wenn Prüflinge in Studium und Weiterbildung Ge- 
legenheit hatten, Interaktionen mit SP zu üben und wenn sie Strategien kennen, um sich adäquat auf diese Prüfungssituation vorzubereiten [Sheen et al., 2015]. In den medizinischen Fächern werden die Leistungen jedes Prüflings an jeder Station einer Parcoursprüfung anhand individueller Leistungsrückmeldungen [Pheister et al., 2017], mithilfe von Checklisten mit dichotomem Antwortformat oder anhand von globalen Ratingskalen eingeschätzt [Hodges et al., 2002; Yudkowsky, 2002]. Für die klinische Psychologie wird der Einsatz etablierter Skalen empfohlen [beispielsweise der Cognitive Therapy Scale oder der Motivational Interviewing Supervision and Training Scale; Edwards et al., 2016]. Da diese Instrumente sehr umfangreich sind und ein detailliertes Urteiler*innentraining erfordern, haben wir die Skala zur Einschätzung Klinischer Gesprächsführung (SEKG) entwickelt, um Basisfertigkeiten der Gesprächsführung (z.B. "Nutzt zur Situation passende Mimik und Gestik") und der therapeutischen Tätigkeit (z.B. "Erarbeitet Inhalte gemeinsam mit dem Patienten") einfach bewerten zu können [Kühne et al., 2020a; erhältlich auf Anfrage von der Korrespondenzautorin].

Weiterhin ist zu bedenken, dass auch für Leistungsbeurteilungen von Prüfer*innen Gütekriterien berichtet werden sollten, da sie deutlich voneinander abweichen können: so bedarf es mehrerer trainierter Prüfer*innen, um zu einer reliablen Kompetenzeinschätzung eines Prüflings zu kommen [Yudkowsky, 2002]. Bezüglich der Anzahl der nötigen Beobachtungen aus SP-Interaktionen pro Prüfling berechneten Imel et al. [2014], dass ca. sechs Beobachtungen notwendig waren, um in ihrem Fall die Adhärenz der Lerner*innen reliabel zu schätzen. Für Sitzungen mit realen Patient*innen waren jedoch deutlich mehr Beobachtungen nötig [Imel et al., 2014]. In einer narrativen Übersichtsarbeit wird von zehn bis vierzehn Rollenspielen mit SP pro Prüfling ausgegangen, um zu einer reliablen Aussage über das Bestehen einer Prüfung kommen zu können [Yudkowsky, 2002]. Parcoursprüfungen sollten also mit einer ausreichenden Anzahl von Stationen zur Kompetenzprüfung geplant werden.

\subsection{Fazit und Ausblick}

Mit dem Gesetz zur Reform der Psychotherapeutenausbildung [2019] werden sich Lehr- und Prüfungsformate in der klinischen Psychologie und Psychotherapie hin zu einer stärkeren Praxisorientierung verändern. SP bieten ein flexibel einsetzbares Instrument für die Lehre und eine Möglichkeit zur reliablen und validen Kompetenzbewertung klinischer Handlungskompetenzen [Hodges et al., 2002; Edwards et al., 2016].

Neben den bereits beschriebenen Anwendungsbereichen sind aber auch weitere Einsatzbereiche und Forschungsthemen möglich. Auf die Zielgruppe angepasst [Hodges et al., 2002] könnten Interaktionen mit
SP genutzt werden, um Weiterbildungskandidat*innen kompetenzbasiert zu supervidieren. Die Supervisionen anschließend zu evaluieren wäre insofern lohnenswert, als dass die Effektivität von Supervision im naturalistischen Setting schwer überprüfbar ist [Willutzki et al., 2015]. Neben dieser eher praxisorientierten Forschung können SP weiterhin für einen experimentellen Vergleich verschiedener Trainings- und Supervisionsmethoden eingesetzt werden [Kühne et al., 2020b]. Schließlich können SP auch in Telefoninterviews im Anschluss an ein Onlinetraining genutzt werden, beispielsweise um zu erfassen, ob Teilnehmer*innen online vermittelte Fertigkeiten (beispielsweise der Motivierenden Gesprächsführung) anwenden können [Edwards et al., 2016].

Auch wenn aktuelle Diskussionen nahelegen, dass die Durchführung der Approbationsprüfung an das IMPP delegiert werden könnte, wird es für die Universitäten relevant sein, die Student*innen bereits während des zur Approbation führenden Masterstudiengangs Psychotherapie auf das neue Prüfungsformat vorzubereiten. Eine Möglichkeit wäre es, bereits den Abschluss einzelner Module, wie zur Vertieften Psychologischen Diagnostik und Begutachtung oder zur Angewandten Psychotherapie vorgeschlagen [PsychThApprO, 2020], mittels praxisnaher Formate und des Einsatzes von SP zu prüfen.

Zusammenfassend bietet der Einsatz von SP aus unserer Sicht großes Potenzial für die zukünftige klinischpsychologische Ausbildung und Forschung. Dabei sollten Gütekriterien (Reliabilität, Validität) durchgängig berücksichtigt und die Effektivität von SP im Vergleich zu anderen aktiven Trainingsbedingungen weiter geprüft werden.

\subsection{Empfehlenswerte Literatur}

Hilfreich für die Gestaltung eigener SP-Programme sind u.a. die Lehrbücher von Voderholzer [2007], in dem auch Beispiele von Fallvignetten abgedruckt sind, und von Peters und Thrien [2018], in dem praktische Hinweise für die Arbeit mit SP in verschiedenen Gesundheitsberufen gegeben werden, sowie der Überblicksartikel von Hodges et al. [2002], der nicht nur Materialien, sondern auch wichtige Erfahrungswerte aufführt.

\section{Statement of Ethics}

Da es sich um eine Übersichtsarbeit handelt, treffen ethische Richtlinien zur Studiendurchführung nicht zu.

\section{Conflict of Interest Statement}

Die Autoren geben an, dass keine Interessenskonflikte bestehen. 


\section{Funding Sources}

Keine externe Förderung.

\section{Literatur}

Adamo G. Simulated and standardized patients in OSCEs: achievements and challenges 1992-2003. Med Teach. 2003 May;25(3):26270.

Approbationsordnung für Psychotherapeutinnen und Psychotherapeuten (PsychThApprO), Bundesgesetzblatt Teil 1 [Internet]. 2020;11:448-83 [cited 2020 Apr 8]. Available from: https://www.bgbl.de/xaver/bgbl/start. $\mathrm{xav}$ ? startbk=Bundesanzeiger_BGBl\& start $=\% 2 \mathrm{~F} \% 2 \mathrm{~F} \% 2 \mathrm{~A} \% 5 \mathrm{~B} \% 40 \mathrm{attr} \_\mathrm{id}=\% 27 \mathrm{bg}$ bl120s0448.pdf\%27\%5D\#__bgbl__\%2F \%2F*\%5B\%40attr_id\%3D\%27bgbl120s0448. pdf\%27\%5D_1586336385782

Ay DS, Weck F, Heinze P, Lang T, Kühne F. Can psychotherapy trainees distinguish standardized patients from real patients? 2020b. Submitted.

Ay DS, Weck F, Kühne F. How to enhance authenticity of simulated patients in psychotherapy training: Results of a randomizedcontrolled study using different role-scripts. 2020a. Submitted.

Ay-Bryson DS, Weck F, Kühne F. Can simulated patient encounters appear authentic? Development and pilot results of a rating instrument based on the portrayal of depressive patients. Train Educ Prof Psychol. 2020. doi: 10.1037/tep0000349.

Barrows HS. An overview of the uses of standardized patients for teaching and evaluating clinical skills. Acad Med. 1993 Jun;68(6):443-51.

Brenner AM. Uses and limitations of simulated patients in psychiatric education. Acad Psychiatry. 2009 Mar-Apr;33(2):112-9.

Coyle B, Miller M, McGowen KR. Using standardized patients to teach and learn psychotherapy. Acad Med. 1998 May;73(5):591-2.

Deutsche Gesellschaft für Psychologie (DGPs) [Internet]. Mögliches Konzept der Psychotherapeutischen Prüfung als inhaltliche Grundlage für die Entwicklung der Approbationsordnung. 2020 [cited 2020 Apr 8]. Available from: https://psychotherapie.dgps.de/fileadmin/user_upload/medien/PsychThG_ Anlage2PsychotherapeutischePru_fung.pdf

Eagles JM, Calder SA, Wilson S, Murdoch JM, Sclare PD. Simulated patients in undergraduate education in psychiatry. Psychiatr Bull. 2007;31(5):187-90.

Eckel J, Merod R, Vogel H, Neuderth S. Einsatz von Schauspielpatienten in den "Psych-" Fächern des Medizinstudiums - Verwendungsmöglichkeiten in der Psychotherapieausbildung? Psychother Psychosom Med Psychol. 2014 Jan;64(1):5-11.

Edwards KS, Parish SJ, Rosen RC, Garvert DW, Spangler SL, Ruzek JI. A standardized patient methodology to assess cognitive-behavioral therapy (CBT) skills performance: Development and testing in a randomized controlled

\section{Author Contributions}

Alle Autor*innen haben maßgeblich zur Konzeption der Arbeit und zur Ausarbeitung des Manuskripts beigetragen. Alle Autor*innen stimmen der Veröffentlichung zu und sind einverstanden, Verantwortung für alle Aspekte der Arbeit zu übernehmen und sicherzustellen, dass Fragen bezüglich der Richtigkeit und Vollständigkeit sämtlicher Teile des Manuskripts angemessen berücksichtigt werden.

trial of web-based training. Train Educ Prof Psychol. 2016;10(3):149-56.

Ehrenthal JC, Dinger U, Montan I, Nikendei C. Neue Lehrformen zur Förderung therapeutischer Kompetenzen. Psychother Dialog. 2019;20(04):64-8.

Fairburn CG, Cooper Z. Therapist competence, therapy quality, and therapist training. Behav Res Ther. 2011 Jun;49(6-7):373-8.

Gesetz zur Reform der Psychotherapeutenausbildung (PsychThG) [Internet]. Bundesgesetzblatt Teil 1. 2019;40:1604-12 [cited 2020 Apr 8]. Available from: https://www.bgbl.de/xav$\mathrm{er} / \mathrm{bgbl} /$ start.xav?startbk=Bundesanzeiger_ BGBl\&start=//*\%5B@attr_id=\%27bgbl119s 1604.pdf\%27\%5D\#_bgbl__\%2F\%2F*\% 5B\%40attr_id\%3D\%27bgbl119s1604. pdf\% $27 \% 5 \mathrm{D}$

Hodges B, Hanson M, McNaughton N, Regehr G; University of Toronto Psychiatric Skills Assessment Project. Creating, monitoring, and improving a psychiatry OSCE: a guide for faculty. Acad Psychiatry. 2002;26(3):134-61.

Imel ZE, Baldwin SA, Baer JS, Hartzler B, Dunn $\mathrm{C}$, Rosengren DB, et al. Evaluating therapist adherence in motivational interviewing by comparing performance with standardized and real patients. J Consult Clin Psychol. 2014 Jun;82(3):472-81.

Kienle R, Peters H [Internet]. Referenzhandbuch: Kommunikation, Interaktion und Teamarbeit. Berlin: Charité; 2016 [cited 2020 Apr 8]. Available from: https://dsfz.charite.de/fileadmin/user_upload/microsites/ohne_AZ/sonstige/studentmentoring/KIT_Referenzhandbuch_DSFZ_Juni_2017.pdf

Kühne F, Ay DS, Otterbeck MJ, Weck F. Standardized patients in clinical psychology and psychotherapy: a scoping review of barriers and facilitators for implementation. Acad Psychiatry. 2018 Dec;42(6):773-81.

Kühne F, Heinze P, Ay DS, Maaß U, Weck F. Development and Validation of a Brief Scale for Assessing Basic Psychotherapeutic Communication Skills. 2020a. Submitted.

Kühne F, Heinze PE, Weck F. Standardized patients in psychotherapy training and clinical supervision: study protocol for a randomized controlled trial. Trials. 2020b Mar;21(1):276.

Muse K, McManus F. A systematic review of methods for assessing competence in cognitive-behavioural therapy. Clin Psychol Rev. 2013 Apr;33(3):484-99.

Nikendei C, Huber J, Ehrenthal JC, Herzog W, Schauenburg H, Schultz JH, et al. Intervention training using peer-role play and standardized patients in psychodynamic psychotherapy trainees. Couns Psychother Res. 2019;19(4):508-22.

Partschefeld E, Strauß B, Geyer M, Philipp S. Simulationspatienten in der Psychotherapieaus- bildung. Psychotherapeut. 2013;58(5):43845.

Partschefeld E, Philipp S, Geyer M, Strauß B [Internet]. Einsatz von SimulationspatientInnen in der Psychotherapieausbildung - Vorstellung eines Lehrkonzepts. DGVT-BV; 2020. Available from: https://www.dgvt-bv.de/newsdetails/?tx_ttnews\%5Btt_news\%5D=3258\&cH $\mathrm{ash}=39 \mathrm{c} 6 \mathrm{aa} 0 \mathrm{a} 4 \mathrm{ca} 339754 \mathrm{ad} 95 \mathrm{a} 0 \mathrm{c} 8 \mathrm{e} 8 \mathrm{c} 4 \mathrm{~b} 9 \mathrm{c}$

Peters T, Thrien C, editors. Simulationspatienten: Handbuch für die Aus- und Weiterbildung in medizinischen- und Gesundheitsberufen. Göttingen: Hogrefe; 2018. https://doi. org/10.1024/85756-000.

Pheister M, Stagno S, Cotes R, Prabhakar D, Mahr F, Crowell A, et al. Simulated patients and scenarios to assess and teach psychiatry residents. Acad Psychiatry. 2017 Feb;41(1):114-7.

Sheen J, McGillivray J, Gurtman C, Boyd L. Assessing the Clinical Competence of Psychology Students Through Objective Structured Clinical Examinations (OSCEs): Student and Staff Views. Aust Psychol. 2015;50(1):51-9.

Universitätsklinikum Hamburg-Eppendorf (UKE) [Internet]. Handbuch für Simulationspatienten am Universitätsklinikum Hamburg-Eppendorf. 2017 [cited 2020 Sept 4]. Available from: https://www.uke.de/dateien/ institute/allgemeinmedizin/dokumente/simpat/handbuch_f\%C3\%BCr_simpats.pdf

Voderholzer U, editor. Lehre im Fach Psychiatrie und Psychotherapie: ein Handbuch. Stuttgart: Kohlhammer; 2007.

Waltz J, Addis ME, Koerner K, Jacobson NS. Testing the integrity of a psychotherapy protocol: assessment of adherence and competence. J Consult Clin Psychol. 1993 Aug;61(4):620-30.

Weck F, Wald A, Kühne F. Erfassung therapeutischer Kompetenzen in der Forschung und Praxis. Psychother Dialog. 2019;20(04):23-7.

Willutzki U, Fydrich T, Strauß B. Aktuelle Entwicklungen in der Psychotherapieausbildung und der Ausbildungsforschung. Psychotherapeut. 2015;60(5):353-64.

Wuendrich MS, Nissen C, Feige B, Philipsen AS, Voderholzer U. Portrayal of psychiatric disorders: are simulated patients authentic? Acad Psychiatry. 2012 Nov;36(6):501-2.

Wündrich M, Schwartz C, Feige B, Lemper D, Nissen C, Voderholzer U. Empathy training in medical students - a randomized controlled trial. Med Teach. 2017 Oct;39(10):1096-8.

Yap K, Bearman M, Thomas N, Hay M. Clinical psychology students' experiences of a pilot objective structured clinical examination. Aust Psychol. 2012;47(3):165-73.

Yudkowsky R. Should we use standardized patients instead of real patients for high-stakes exams in psychiatry? Acad Psychiatry. 2002; 26(3):187-92 